\title{
Endometrioid Stromal Sarcoma of the Cervix
}

National Cancer Institute

\section{Source}

National Cancer Institute. Endometrioid Stromal Sarcoma of the Cervix. NCI Thesaurus.

Code $C 40220$.

A rare sarcoma that arises from the cervix. This category includes low grade

endometrioid stromal sarcoma and undifferentiated endocervical sarcoma. 